Old Dominion University

ODU Digital Commons

\title{
Chiral Effective Field Theory Calculations of Weak Transitions in Light Nuclei
}

\author{
G.B. King \\ L. Andreoli \\ S. Pastore \\ M. Piarulli \\ R. Schiavilla \\ Old Dominion University, rschiavi@odu.edu
}

See next page for additional authors

Follow this and additional works at: https://digitalcommons.odu.edu/physics_fac_pubs

Part of the Nuclear Commons

\section{Original Publication Citation}

King, G. B., Andreoli, L., Pastore, S., Piarulli, M., Schiavilla, R., Wiringa, R. B., Carlson, J., \& Gandolfi, S. (2020). Chiral effective field theory calculations of weak transitions in light nuclei. Physical Review $C$, 102(2), 13 pp., Article 025501. https://doi.org/10.1103/PhysRevC.102.025501

This Article is brought to you for free and open access by the Physics at ODU Digital Commons. It has been accepted for inclusion in Physics Faculty Publications by an authorized administrator of ODU Digital Commons. For more information, please contact digitalcommons@odu.edu. 


\section{Authors}

G.B. King, L. Andreoli, S. Pastore, M. Piarulli, R. Schiavilla, R.B. Wiringa, J. Carlson, and S. Gandolfi 


\title{
Chiral effective field theory calculations of weak transitions in light nuclei
}

\author{
G. B. King $\odot,{ }^{1}$ L. Andreoli $\odot,{ }^{1}$ S. Pastore $\odot,{ }^{1,2}$ M. Piarulli, ${ }^{1}$ R. Schiavilla $\odot,{ }^{3,4}$ R. B. Wiringa, ${ }^{5}$ J. Carlson,${ }^{6}$ and S. Gandolfi $\oplus^{6}$ \\ ${ }^{1}$ Department of Physics, Washington University in Saint Louis, Saint Louis, Missouri 63130, USA \\ ${ }^{2}$ McDonnell Center for the Space Sciences at Washington University in St. Louis, Missouri 63130, USA \\ ${ }^{3}$ Department of Physics, Old Dominion University, Norfolk, Virginia 23529, USA \\ ${ }^{4}$ Theory Center, Jefferson Lab, Newport News, Virginia 23606, USA \\ ${ }^{5}$ Physics Division, Argonne National Laboratory, Argonne, Illinois 60439, USA \\ ${ }^{6}$ Theoretical Division, Los Alamos National Laboratory, Los Alamos, New Mexico 87545, USA
}

(Received 10 April 2020; accepted 22 June 2020; published 20 August 2020)

\begin{abstract}
We report quantum Monte Carlo calculations of weak transitions in $A \leqslant 10$ nuclei, based on the Norfolk two- and three-nucleon chiral interactions, and associated one- and two-body axial currents. We find that the contribution from two-body currents is at the $2-3 \%$ level, with the exception of matrix elements entering the rates of ${ }^{8} \mathrm{Li},{ }^{8} \mathrm{~B}$, and ${ }^{8} \mathrm{He} \beta$ decay. These matrix elements are suppressed in impulse approximation based on the (leading order) Gamow Teller transition operator alone; two-body currents provide a $20-30 \%$ correction, which is, however, insufficient to bring theory in agreement with experimental data. For the other transitions, the agreement with the data is satisfactory, and the results exhibit a negligible to mild model dependence when different combinations of Norfolk interactions are utilized to construct the nuclear wave functions. We report a complete study of two-body weak transition densities which reveals the expected universal behavior of two-body currents at short distances throughout the range of $A=3$ to $A=10$ systems considered here.
\end{abstract}

DOI: 10.1103/PhysRevC.102.025501

\section{INTRODUCTION}

In this work, we present quantum Monte Carlo (QMC) calculations, including both variational Monte Carlo (VMC) and Green's function Monte Carlo (GFMC) calculations, of Gamow-Teller (GT) matrix elements entering $\beta$-decay and electron-capture rates in $A=3-10$ nuclei. These observables are experimentally known (in most cases) at the subpercentage level, and are used here primarily to validate our microscopic theoretical modeling of the nucleus as a system of nucleons interacting with each other via effective interactions, and with electroweak probes via effective currents. Specifically, this modeling is based on local two- and three-nucleon interactions formulated in configuration space, and derived from a chiral effective field theory ( $\chi$ EFT) that retains, in addition to nucleons and pions, $\Delta$ isobars as explicit degrees of freedom [1-4]. They are referred to below as the Norfolk interactions and are denoted as $\mathrm{NV} 2+3$. Accompanying these interactions are one- and two-body axial currents-local in configuration space-derived within the same $\chi$ EFT formulation [3-5]. In the calculations to follow, we include up to tree-level contributions at next-to-next-to-next-to-leading order (N3LO) in the chiral expansion, and disregard subleading corrections involving loops and higher order contact terms.

An analogous study was recently reported by some of the present authors in Ref. [6]. There, the QMC calculations were based on the Argonne- $v_{18}$ (AV18) two-nucleon [7] and Illinois-7 (IL-7) three-nucleon [8] interactions, in combination with the axial currents of Ref. [5]. We found agreement with the experimental Gamow-Teller matrix elements at the $\approx 2 \%$ level for the $A=6$ and 7 systems, and at the $\approx 10 \%$ level in ${ }^{10} \mathrm{C}$. The large uncertainty in the $A=10$ transition is primarily systematic and results from the narrow energy separation between the first two $J^{\pi}=1^{+}$states in ${ }^{10} \mathrm{~B}$, which makes it hard to precisely disentangle them [6]. The study of Ref. [6] found that two-body currents generate an additive contribution of the order of $\approx 3 \%$ and concluded that the agreement with the data is mainly attributable to the use of fully correlated nuclear wave functions, rather than two-body effects in the currents.

In the meantime, no-core shell-model calculations of weak matrix elements based on chiral interactions and currents [9] found the sign of the overall correction generated by two-body currents to be opposite to that obtained in Ref. [6] for the same systems (but in agreement with a hybrid calculation of the $A=6$ decay reported in Ref. [10]). This discrepancy was attributed to the hybrid nature of the calculation of Ref. [6], i.e., to the mismatch between the two- and three-body correlations implemented to construct the nuclear wave functions-and induced by the AV18 and IL7-and those entering the axial currents which were instead derived from $\chi$ EFT.

In this work, by reexamining the evaluation of these weak matrix elements with the NV2+3 chiral interactions [1-4], in combination with consistent chiral axial currents at tree level [5], we aim to address and explore the aforementioned claim. We investigate the sensitivity of the calculated matrix elements with respect to different choices of regulators and to different strategies adopted to constrain the three-body Norfolk interactions (NV3). This latter aspect is important in order to understand the interplay between these interactions 
and the axial currents, since the strength of the contact current and that of the three-body interaction of one-pion-range are rigorously related to each other by the symmetries imposed in the $\chi$ EFT formulation [11-13].

This study has several merits. First, we report new GFMC results of the energy spectra of $A \leqslant 10$ nuclei based on two classes of $\mathrm{NV} 2+3$ interactions. In addition to the systems studied in Ref. [6], we study weak transitions in $A=8$ nuclei where we find that two-body axial currents provide a large correction to the one-body results. Finally, we provide the first calculations of two-body weak transition densities which shed light on the role of short-range physics in these observables.

Searches for physics beyond the standard model (BSM) via $\beta$ decay are the focus of current and planned experimental programs carried out at the Facility for Rare Isotope Beams (FRIB), the the University of Washington, and Argonne National Laboratory (see, e.g., Ref. [14] and references therein). Among the targets under consideration are ${ }^{6} \mathrm{He},{ }^{8} \mathrm{Li},{ }^{8} \mathrm{~B}$, and ${ }^{10} \mathrm{C}$. A systematic study of axial-current matrix elements in these systems is a prerequisite for all further investigations and BSM searches in $\beta$ decay. $\beta$ decays are ideal processes to assess the validity of the dynamical inputs of $a b$ inito calculations, namely many-body correlations and weak currents. The latter also impact calculations of neutrinoless double $\beta$ decay matrix elements, whose knowledge is critical to the neutrinoless double $\beta$ decay experimental program [15].

This paper is structured as follows: a brief review of the QMC computational method and Norfolk interactions is given in Secs. II and III. The many-body axial currents used in this work are reported in Sec. IV. Results and conclusions are provided in Secs. V and VI.

\section{QUANTUM MONTE CARLO METHOD}

The quantum Monte Carlo methods used in this study have been described in detail in several review articles, the most recent of which being Refs. [16,17]. Here we sketch the computational procedure and refer the interested reader to Refs. [16,17] and references therein.

We seek accurate solutions of the many-nucleon Schrödinger equation

$$
H \Psi\left(J^{\pi} ; T, T_{z}\right)=E \Psi\left(J^{\pi} ; T, T_{z}\right),
$$

where $J^{\pi}$ are the total angular momentum and parity of the state and $T$ and $T_{z}$ are the total isospin and its projection, respectively. We use the Hamiltonian

$$
H=\sum_{i} K_{i}+\sum_{i<j} v_{i j}+\sum_{i<j<k} V_{i j k},
$$

where $K_{i}$ is the nonrelativistic kinetic energy and $v_{i j}$ and $V_{i j k}$ are the NV2 and NV3 local chiral interactions [1-4], collectively denoted as NV2 +3 .

The VMC trial function $\Psi_{V}\left(J^{\pi} ; T, T_{z}\right)$ for a given nucleus is constructed from products of two- and three-body correlation operators acting on an antisymmetric single-particle state of the appropriate quantum numbers. The correlation operators are designed to reflect the influence of the interactions at short distances, while appropriate boundary conditions are imposed at long range [18-21]. The $\Psi_{V}\left(J^{\pi} ; T, T_{z}\right)$ contains variational parameters that are adjusted to minimize the expectation value

$$
E_{V}=\frac{\left\langle\Psi_{V}|H| \Psi_{V}\right\rangle}{\left\langle\Psi_{V} \mid \Psi_{V}\right\rangle} \geqslant E_{0},
$$

which is evaluated by Metropolis Monte Carlo integration [22]. The lowest value for $E_{V}$ is then taken as the approximate ground-state energy of the exact lowest eigenvalue of $H, E_{0}$, for the specified quantum numbers.

A good trial wave function is given by

$$
\left|\Psi_{V}\right\rangle=\mathcal{S} \prod_{i<j}^{A}\left[1+U_{i j}+\sum_{k \neq i, j}^{A} \tilde{U}_{i j k}^{T N I}\right]\left|\Psi_{J}\right\rangle .
$$

The Jastrow wave function $\Psi_{J}$ is fully antisymmetric and has the $\left(J^{\pi} ; T, T_{z}\right)$ quantum numbers of the state of interest, while $U_{i j}$ and $\widetilde{U}_{i j k}^{T N I}$ are two- and three-body correlation operators.

The GFMC method [16] improves on the VMC wave functions by acting on $\Psi_{V}$ with the operator exp $\left[-\left(H-E_{0}\right) \tau\right]$. The operator is applied in a sequence of small imaginary-time steps $\Delta \tau$ to produce a propagated wave function

$$
\Psi(\tau)=e^{-\left(H-E_{0}\right) \tau} \Psi_{V}=\left[e^{-\left(H-E_{0}\right) \Delta \tau}\right]^{n} \Psi_{V} .
$$

Obviously $\Psi(\tau=0)=\Psi_{V}$ and $\Psi(\tau \rightarrow \infty)=\Psi_{0}$. Quantities of interest are evaluated in terms of a "mixed" expectation value between $\Psi_{V}$ and $\Psi(\tau)$ :

$$
\langle O(\tau)\rangle_{M}=\frac{\left\langle\Psi(\tau)|O| \Psi_{V}\right\rangle}{\left\langle\Psi(\tau) \mid \Psi_{V}\right\rangle},
$$

where the operator $O$ acts on the trial function $\Psi_{V}$. The desired expectation values would, of course, have $\Psi(\tau)$ on both sides; by writing $\Psi(\tau)=\Psi_{V}+\delta \Psi(\tau)$ and neglecting terms of order $[\delta \Psi(\tau)]^{2}$, we obtain the approximate expression

$$
\begin{aligned}
\langle O(\tau)\rangle & =\frac{\langle\Psi(\tau)|O| \Psi(\tau)\rangle}{\langle\Psi(\tau) \mid \Psi(\tau)\rangle} \\
& \approx\langle O(\tau)\rangle_{M}+\left[\langle O(\tau)\rangle_{M}-\langle O\rangle_{V}\right],
\end{aligned}
$$

where $\langle O\rangle_{\mathrm{V}}$ is the variational expectation value.

For off-diagonal matrix elements required by the transitions we are interested here, the generalized mixed estimate is given by the expression

$$
\begin{gathered}
\frac{\left\langle\Psi^{f}(\tau)|O| \Psi^{i}(\tau)\right\rangle}{\sqrt{\left\langle\Psi^{f}(\tau) \mid \Psi^{f}(\tau)\right\rangle} \sqrt{\left\langle\Psi^{i}(\tau) \mid \Psi^{i}(\tau)\right\rangle}} \\
\approx\langle O(\tau)\rangle_{M_{i}}+\langle O(\tau)\rangle_{M_{f}}-\langle O\rangle_{V},
\end{gathered}
$$

where

$$
\langle O(\tau)\rangle_{M_{f}}=\frac{\left\langle\Psi^{f}(\tau)|O| \Psi_{V}^{i}\right\rangle}{\left\langle\Psi^{f}(\tau) \mid \Psi_{V}^{f}\right\rangle} \sqrt{\frac{\left\langle\Psi_{V}^{f} \mid \Psi_{V}^{f}\right\rangle}{\left\langle\Psi_{V}^{i} \mid \Psi_{V}^{i}\right\rangle}},
$$

and $\langle O(\tau)\rangle_{M_{i}}$ is defined similarly. For more details see Eqs. (19)-(24) and the accompanying discussions in Ref. [23].

\section{NORFOLK INTERACTION MODELS}

We base our calculations of weak transitions in $A=6-$ 10 on the local NV2 and NV3 interactions developed in Refs. [1-4]. The NV2 model has been derived from a $\chi$ EFT 
TABLE I. Values of (fixed) low energy constants (LECs) used in this work: $\tilde{g}_{A}$ and $h_{A}$ are adimensional, $F_{\pi}=2 f_{\pi}$ is given in $\mathrm{MeV}$, and the remaining LECs are given in $\mathrm{GeV}^{-1}$. See text for explanation.

\begin{tabular}{cccccccc}
\hline \hline$\tilde{g}_{A}$ & $h_{A}$ & $F_{\pi}$ & $c_{1}$ & $c_{2}$ & $c_{3}$ & $c_{4}$ & $b_{3}+b_{8}$ \\
\hline 1.29 & 2.74 & 184.80 & -0.57 & -0.25 & -0.79 & 1.33 & 1.40 \\
\hline \hline
\end{tabular}

that uses pions, nucleons, and $\Delta$ 's as fundamental degrees of freedom. It consists of a long-range part, $v_{i j}^{\mathrm{L}}$, mediated by one- and two-pion exchanges, and a short-range part, $v_{i j}^{S}$, described in terms of contact interactions with strengths specified by unknown low-energy constants (LECs). The strength of the long-range part is fully determined by the nucleon and nucleon-to- $\Delta$ axial coupling constants $g_{A}$ and $h_{A}$, the pion-decay amplitude $F_{\pi}$, and the LECs $c_{1}, c_{2}, c_{3}$, $c_{4}$, and $b_{3}+b_{8}$, constrained by reproducing $\pi N$ scattering data [24]. The LECs entering the contact interactions are fixed by fitting nucleon-nucleon scattering data from the most recent and up-to-date database collected by the Granada group [25-27]. The value for $h_{A}$ is taken from the large $N_{c}$ expansion or strong-coupling model [28]. The value of the nucleon axial coupling constant used to construct the nucleon-nucleon interaction accounts for the Goldberger-Treiman discrepancy $[29,30]$ and, to distinguish it from the experimental value of $g_{A}=1.2723$ (23) [31] entering the axial currents, we denote it with $\tilde{g}_{A}$. For completeness, Tables I and II report the values of these constants, along with the pion and nucleon masses, the $\Delta$-nucleon mass difference, the electron mass, and the fine structure constant $\alpha$ used in the NV2 interactions (these last two characterize the electromagnetic part of the NV2s [1]).

The contact terms are implemented using a Gaussian representation of the three dimensional $\delta$ function, with $R_{S}$ denoting the Gaussian parameter [1-4]. The pion-range operators are strongly singular at short range in configuration space and are regularized by a radial function characterized by a cutoff $R_{L}$ [1-4]. There are two classes (I and II) of NV2s, differing only in the range of energy over which they are fitted to the database-class I up to $125 \mathrm{MeV}$ and class II up to $200 \mathrm{MeV}$. For each class, two combinations of short- and long-range regulators have been used, namely $\left(R_{S}, R_{L}\right)=(0.8,1.2) \mathrm{fm}$ (models NV2-Ia and NV2-IIa) and $\left(R_{S}, R_{L}\right)=(0.7,1.0) \mathrm{fm}$ (models NV2-Ib and NV2-IIb). Class I (II) fits about 2700 (3700) data points with a $\chi^{2} /$ datum $\lesssim 1.1(\lesssim 1.4)[1,2]$.

The NV2 models were found to provide insufficient attraction in GFMC calculations of the binding energies of light nuclei [2]. To remedy this shortcoming, a consistent three-body interaction was constructed up to N2LO in the chiral expansion. It consists of a long-range part mediated

TABLE II. Values of charged and neutral pion masses, proton and neutron masses, $\Delta$-nucleon mass difference, and electron mass (all in $\mathrm{MeV}$ ), and of the (adimensional) fine structure constant $\alpha$. Note that $\hbar c$ is taken as $197.32697 \mathrm{MeV}$ fm.

\begin{tabular}{llllllll}
\hline \hline$m_{\pi_{0}}$ & & $m_{\pi_{ \pm}}$ & $M_{n}$ & $M_{p}$ & $m_{\Delta N}$ & $m_{e}$ & $\alpha^{-1}$ \\
\hline
\end{tabular}

$\begin{array}{lllllll}134.9766 & 139.5702 & 939.56524 & 938.27192 & 293.1 & 0.510999 & 137.03599\end{array}$
TABLE III. Adimensional $c_{D}$ and $c_{E}$ values of the contact terms in the NV3 interactions obtained from fits to (i) the $n d$ scattering length and trinucleon binding energies [37] and (ii) the central value of the ${ }^{3} \mathrm{H}$ GT matrix element and the trinucleon binding energies (starred values) [4]. The adimensional $z_{0}$ values are obtained using the relation given in Eq. (16).

\begin{tabular}{|c|c|c|c|c|}
\hline & $\mathrm{Ia}\left(\mathrm{Ia}^{*}\right)$ & $\mathrm{Ib}\left(\mathrm{Ib}^{*}\right)$ & IIa $\left(\mathrm{IIa}^{*}\right)$ & $\mathrm{IIb}\left(\mathrm{IIb}^{*}\right)$ \\
\hline \multicolumn{3}{|c|}{$c_{D} 3.666(-0.635)-2.061(-4.71)$} & $1.278(-0.6$ & $-4.480(-5.25)$ \\
\hline \multicolumn{3}{|c|}{$c_{E}-1.638(-0.090)-0.982(0.55)$} & $-1.029(-0.3$ & $-0.412(0.05)$ \\
\hline$z_{0}$ & $0.090(1.035)$ & $2.013(2.881)$ & $0.615(1.03)$ & $2.806(3.059)$ \\
\hline
\end{tabular}

by two-pion exchange and a short-range part parametrized in terms of two contact interactions [32,33] proportional to the LECs $c_{D}$ and $c_{E}$. These LECs have been obtained by fitting either observables that involve exclusively strong interactions [34-37] or a combination of observables that involve both strong and weak interactions $[4,12,38]$. This last strategy is feasible because of the relation established in $\chi$ EFT [11] that links $c_{D}$ with the LECs entering the contact axial current at N3LO $[12,13,38]$ (see next section for details).

In Ref. [37], $c_{D}$ and $c_{E}$ were determined by simultaneously reproducing the experimental trinucleon groundstate energies and $n d$ doublet scattering length. These firstgeneration $\mathrm{NV} 2+3$ interactions, denoted with $\mathrm{NV} 2+3-\mathrm{Ia} / \mathrm{b}$ and NV2+3-IIa/b, have been implemented in both VMC and GFMC codes and used to study static properties of light nuclei $[2,17,37,39-41]$ and in auxiliary-field diffusion Monte Carlo (AFDMC) [42], Brueckner-Bethe-Goldstone (BBG) $[43,44]$, and Fermi hypernetted chain/single-operator chain (FHNC/SOC) $[45,46]$ approaches to investigate the equation of state of neutron matter $[47,48]$.

In more recent work [4], $c_{D}$ and $c_{E}$ were constrained by fitting, in addition to the trinucleon energies, the empirical value of the GT matrix element in tritium $\beta$ decay. These second-generation $\mathrm{NV} 2+3$ interactions were designated as $\mathrm{NV} 2+3-\mathrm{Ia}^{*} / \mathrm{b}^{*}$ and NV2+3-IIa* $/ \mathrm{b}^{*}$. These two different procedures for fixing $c_{D}$ and $c_{E}$ produced rather different values for these LECs. They are reported in Table III.

\section{IV. $\chi$ EFT AXIAL CURRENTS}

Many-body axial currents have been first examined within $\chi$ EFT by Park and collaborators in Ref. [49]. In that work, the authors retained pions and nucleons in their effective theory and calculated the two-body axial currents up to oneloop terms. The derivation neglected, for example, pion-pole contributions. More recently, two-body axial currents with pions and nucleons have been derived by the Bonn group [50] using the unitary transformation method, and by the JLabPisa group using time-ordered perturbation theory $[3,5]$. The two derivations differ in the treatment of reducible diagrams. When calculating box diagrams entering the electromagnetic charge and current operators [51-55], the two methods lead to results that are in agreement. However, as discussed at length in Refs. $[3,56]$, the two groups find different results for the box diagrams in the two-body axial current operator at N4LO. The numerical impact of this difference has been investigated in 
(a)

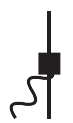

(b)

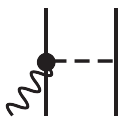

(d) $\sqrt{1}$
LO

\section{N2LO}

(c)

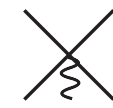

(e)
FIG. 1. Diagrams illustrating the contributions to the axial current up to N3LO used in this work. Nucleons, $\Delta$ isobars, pions, and external fields are denoted by solid, thick-solid, dashed, and wavy lines, respectively. The square in panel (b) represents relativistic corrections, while the dot in panels (d) denotes a vertex induced by subleading terms in the $\pi$-nucleon chiral Lagrangian [3].

Refs. [3,4], where both the JLab-Pisa and Bonn versions of the N4LO current operators have been implemented to calculate the GT matrix element in triton $\beta$ decay. In those studies, it was found that the corrections generated by the JLab-Pisa and Bonn N4LO operators are qualitatively in agreement (they both quench the GT matrix element at leading order), and provide, respectively, a $\approx 6 \%$ and $\approx 4 \%$ contribution to the total GT matrix element.

Here we consider two-body axial currents derived within the same $\chi$ EFT used to construct the NV2+3 interactions [4]. Moreover, we base our calculations on tree-level corrections only, and disregard the (problematic) N4LO loop contributions discussed above. This choice is advantageous also because it allows for a clearer comparison with the nocore shell model and coupled-cluster calculations of Ref. [9], which are also based on tree-level axial currents alone (albeit derived in a $\Delta$-less $\chi \mathrm{EFT}$ ). Corrections at N4LO in the present formulation are, in practice, subsumed in the LECs of the theory, which have been determined by fits to experimental data.

Before moving on to a (brief) discussion of these axial currents, it is worthwhile pointing out that many-body corrections to leading one-body transition operators have been shown to be crucial for providing a quantitatively successful description of many nuclear electroweak observables [57], such as nuclear electromagnetic form factors [58-61], low-energy electroweak transitions $[6,52,53,62-65]$, and electroweak scattering [66]. They have also been used in studies of double $\beta$ decay matrix elements $[39,67-69]$.

The N3LO axial currents used in this work are represented diagrammatically Fig. 1 . We refer the interested reader to Refs. [4,5] for additional details and explicit expressions of the operators; here we only note that we do not show diagrams that lead to vanishing contributions as well as pion-pole terms which give negligible corrections to the matrix elements under study.

The LO term, which scales as $Q^{-3}$ in the power counting ( $Q$ denotes generically a low-momentum scale), is shown in
Fig. 1(a) and reads

$$
\mathbf{j}_{5, a}^{\mathrm{LO}}(\mathbf{q})=-\frac{g_{A}}{2} \tau_{i, a} \boldsymbol{\sigma}_{i} e^{i \mathbf{q} \cdot \mathbf{r}_{i}},
$$

where $g_{A}$ is the nucleon axial coupling constant $\left(g_{A}=1.2723\right.$ [31]), $f_{\pi}$ is the pion-decay amplitude $\left(f_{\pi}=92.4 \mathrm{MeV}\right), \sigma_{i}$ and $\boldsymbol{\tau}_{i}$ are the spin and isospin Pauli matrices of nucleon $i, \mathbf{q}$ is the external field momentum, $\mathbf{r}_{i}$ is the position of nucleon $i$, and the subscript $a$ specifies the isospin component ( $a=$ $x, y, z)$.

At N2LO there are two contributions (scaling as $Q^{-1}$ ). The first one is a relativistic correction to the single-nucleon operator at LO and is diagrammatically illustrated in Fig. 1(b), while the second involves the excitation of a nucleon into a $\Delta$ by pion exchange, as illustrated in Fig. 1(c). In the tables and figures below, we will denote these two contributions with N2LO-RC and N2LO- $\Delta$, respectively. We use the same notation introduced in Ref. [4] and write the cumulative N2LO contribution as

$$
\mathbf{j}_{5, a}^{\mathrm{N} 2 \mathrm{LO}}(\mathbf{q})=\mathbf{j}_{5, a}^{\mathrm{N} 2 \mathrm{LO}}(\mathbf{q} ; \mathrm{RC})+\mathbf{j}_{5, a}^{\mathrm{N} 2 \mathrm{LO}}(\mathbf{q} ; \Delta) .
$$

At N3LO (or $Q^{0}$ in the chiral expansion), there is a term of one-pion range illustrated in Fig. 1(d), and a contact term shown in Fig. 1(e), which together give the following N3LO correction

$$
\mathbf{j}_{5, a}^{\mathrm{N} 3 \mathrm{LO}}(\mathbf{q})=\mathbf{j}_{5, a}^{\mathrm{N} 3 \mathrm{LO}}(\mathbf{q} ; \mathrm{OPE})+\mathbf{j}_{5, a}^{\mathrm{N} 3 \mathrm{LO}}(\mathbf{q} ; \mathrm{CT}) .
$$

We will denote the individual terms with N3LO-OPE and N3LO-CT, respectively.

The configuration-space expressions of these currents are given in Eqs. (2.7) - (2.10) of Ref. [4]. Here we limit ourselves to report the expression of the N3LO contact term used in this work to explicitly show the relation between the LECs entering this axial current and the LEC $c_{D}$ in the three-nucleon interaction. In $r$ space the N3LO-CT current reads

$$
\mathbf{j}_{5, a}^{\mathrm{N} 3 \mathrm{LO}}(\mathbf{q} ; \mathrm{CT})=z_{0} e^{i \mathbf{q} \cdot \mathbf{R}_{i j}} \frac{e^{-z_{i j}^{2}}}{\pi^{3 / 2}}\left(\boldsymbol{\tau}_{i} \times \boldsymbol{\tau}_{j}\right)_{a}\left(\boldsymbol{\sigma}_{i} \times \boldsymbol{\sigma}_{j}\right),
$$

where

$$
\mathbf{R}_{i j}=\left(\mathbf{r}_{i}+\mathbf{r}_{j}\right) / 2, \quad z_{i j}=r_{i j} / R_{S},
$$

and $r_{i j}$ is the interparticle distance. The $\delta$ function in the contact axial current has been smeared by replacing it with a Gaussian cutoff of range $R_{S}$,

$$
C_{R_{S}}\left(r_{i j}\right)=\frac{1}{\pi^{3 / 2} R_{S}^{3}} e^{-\left(r_{i j} / R_{S}\right)^{2}},
$$

as previously done for the contactlike terms of the NV2 interactions. The adimensional LEC $z_{0}$ (reported in Table III) is given by

$$
\begin{aligned}
z_{0}= & \frac{g_{A}}{2} \frac{m_{\pi}^{2}}{f_{\pi}^{2}} \frac{1}{\left(m_{\pi} R_{S}\right)^{3}}\left[-\frac{m_{\pi}}{4 g_{A} \Lambda_{\chi}} c_{D}\right. \\
& \left.+\frac{m_{\pi}}{3}\left(c_{3}+2 c_{4}\right)+\frac{m_{\pi}}{6 m}\right],
\end{aligned}
$$

where $c_{D}$ is the LEC multiplying one of the contact terms in the three-nucleon interaction [33] given in Table III, $\Lambda_{\chi}=$ $1 \mathrm{GeV}$ is the chiral symmetry breaking scale, $c_{3}$ and $c_{4}$ are 
TABLE IV. GFMC predictions for $A \leqslant 10$ nuclear states studied in this work, compared to experimental values [70-72]. The ${ }^{4} \mathrm{He}$ ground-state energy predictions are included in addition to the states studied in this work. Numbers in parentheses are statistical errors for the GFMC calculations; experimental errors, being negligible, are not indicated. The dominant spatial symmetry (s.s.) of the nuclear wave function is given in the second column.

\begin{tabular}{lclll}
\hline \hline${ }^{A} Z\left(J^{\pi} ; T\right)$ & s.s. & \multicolumn{2}{c}{$E(\mathrm{MeV})$} & \\
\cline { 3 - 4 } & & $\mathrm{Ia}$ & $\mathrm{Ia}$ & Expt. \\
\hline${ }^{4} \mathrm{He}\left(0^{+} ; 0\right)$ & {$[4]$} & $-28.24(3)$ & $-28.21(4)$ & -28.30 \\
${ }^{6} \mathrm{Li}\left(1^{+} ; 0\right)$ & {$[42]$} & $-31.97(6)$ & $-31.06(8)$ & -31.99 \\
${ }^{6} \mathrm{He}\left(0^{+} ; 0\right)$ & {$[42]$} & $-29.32(4)$ & $-28.46(5)$ & -29.27 \\
${ }^{7} \mathrm{Li}\left(\frac{3}{2}^{-} ; \frac{1}{2}\right)$ & {$[43]$} & $-39.25(15)$ & $-38.27(14)$ & -39.25 \\
${ }^{7} \mathrm{Li}\left(\frac{1}{2}^{-} ; \frac{1}{2}\right)$ & {$[43]$} & $-39.18(15)$ & $-37.66(15)$ & -38.76 \\
${ }^{7} \mathrm{Be}\left(\left(^{\frac{3}{2}} ; \frac{1}{2}\right)\right.$ & {$[43]$} & $-37.75(8)$ & $-36.56(10)$ & -37.60 \\
${ }^{8} \mathrm{He}\left(0^{+} ; 2\right)$ & {$[422]$} & $-31.33(7)$ & $-28.53(6)$ & -31.40 \\
${ }^{8} \mathrm{Li}\left(2^{+} ; 1\right)$ & {$[431]$} & $-41.59(10)$ & $-38.89(7)$ & -41.28 \\
${ }^{8} \mathrm{Li}\left(1^{+} ; 1\right)$ & {$[431]$} & $-40.59(7)$ & $-37.78(7)$ & -40.30 \\
${ }^{8} \mathrm{~B}\left(2^{+} ; 1\right)$ & {$[431]$} & $-37.87(8)$ & $-35.63(8)$ & -37.74 \\
${ }^{8} \mathrm{Be}\left(2^{+} ; 0\right)$ & {$[44]$} & $-54.07(7)$ & $-53.16(11)$ & -53.47 \\
${ }^{10} \mathrm{~B}\left(1^{+} ; 0\right)$ & {$[442]$} & $-64.61(41)$ & $-60.46(30)$ & -64.03 \\
${ }^{10} \mathrm{C}\left(0^{+} ; 1\right)$ & {$[442]$} & $-61.01(50)$ & $-56.65(22)$ & -60.32 \\
\hline \hline
\end{tabular}

given in Table I, and $m$ and $m_{\pi}$ are the average nucleon and pion masses. It has recently been realized [13] that the relation between $z_{0}$ and $c_{D}$ had been given erroneously in the original reference [12], a - sign and a factor $1 / 4$ were missing in the term proportional to $c_{D}$.

\section{RESULTS}

The GFMC energies of the nuclei of interest (as well as ${ }^{4} \mathrm{He}$ ) calculated using the NV2+3-Ia and NV2+3-Ia* models are listed in Table IV along with the dominant spatial symmetry (s.s.) of the variational wave functions [73]. The energies are obtained using $\approx 80000$ walkers, and are all well converged by 30 unconstrained steps [74]. All the GFMC results presented in this article (but for the two cases discussed below) are averages over the imaginary time $\tau$ from 0.2 to $0.82 \mathrm{MeV}^{-1}$. Results obtained with the NV2+3-Ia interaction are in statistical agreement with those published in Ref. [37] based on the same nuclear Hamiltonian. Model NV2+3-Ia leads to predictions that are in excellent agreement with the data. We also report for the first time results based on the second generation of $\mathrm{NV} 2+3$ interactions, specifically model $\mathrm{NV} 2+3-\mathrm{Ia}^{*}$, whose three-nucleon interaction has been constrained by fits to the experimental trinucleon binding energies and tritium GT matrix element. Results obtained with the $\mathrm{NV} 2+3-\mathrm{Ia}^{*}$ Hamiltonian display a somewhat less satisfactory agreement with the experimental data, but still less than $4 \%$ away from them.

A typical imaginary-time evolution of the GFMC transition matrix elements is shown in Fig. 2. As can be seen, there is a rapid drop of $3 \%$ from the initial VMC estimate at $\tau=0$ that reaches a stable value around $0.2 \mathrm{MeV}^{-1}$. The results for all transitions presented in this article are averages over $\tau$ from



FIG. 2. Propagation of the ${ }^{6} \mathrm{He} \rightarrow{ }^{6} \mathrm{Li}$ transition matrix element as function of imaginary time $\tau$, based on the NV2+3-Ia Hamiltonian. Results with the one-body current at LO and currents beyond $\mathrm{LO}$ are indicated with $1 \mathrm{~b}$ and $2 \mathrm{~b}$, respectively. Dashed and solid lines represent central values and associated error bars. Black dashed and solid lines denote the VMC results. See text for further explanations.

0.2 to $0.82 \mathrm{MeV}^{-1}$, as indicated by the dashed lines, with statistical errors denoted by the solid lines. The calculations of weak transitions involving the $\left(J^{\pi}, T\right)=\left(2^{+}, 0\right)$ state of ${ }^{8} \mathrm{Be}$ and the ground state of ${ }^{8} \mathrm{~B}$ are treated differently. For these two states, we observe that the binding energy, magnitude of the quadrupole moment, and point-proton radius all increase monotonically as the imaginary time increases. This can be appreciated in Fig. 3 where we show the point-proton radii of the two nuclear states. We interpret this behavior as an indication that the resonant excited state of ${ }^{8} \mathrm{Be}$ is dissolving into two separated $\alpha$ 's, while ${ }^{8} \mathrm{~B}$ is breaking into $p+{ }^{7} \mathrm{Be}$. In the case of ${ }^{8} \mathrm{Be}$, this issue has been addressed already in Refs. [64,65,74]. Here, we use similar techniques to treat these systems and extract matrix elements from the GFMC data. In particular, we note that the ground-state energy of ${ }^{8} \mathrm{Be}$ drops very quickly as the imaginary time increases and reaches stability around $\tau \sim 0.1 \mathrm{MeV}^{-1}$. The transition matrix elements involving the two dissolving states have been determined assuming that, also for these states, $\tau \approx 0.1 \mathrm{MeV}^{-1}$ is the point at which spurious contaminations in the nuclear wave functions have been removed by the GFMC propagation. We then average in a small interval around this point, typically between 0.06 and $0.14 \mathrm{MeV}^{-1}$. Calculations involving these two systems are clearly affected by a systematic error. To have a rather rough estimate of this error, we study the sensitivity of the extracted matrix elements with respect to variations in the 


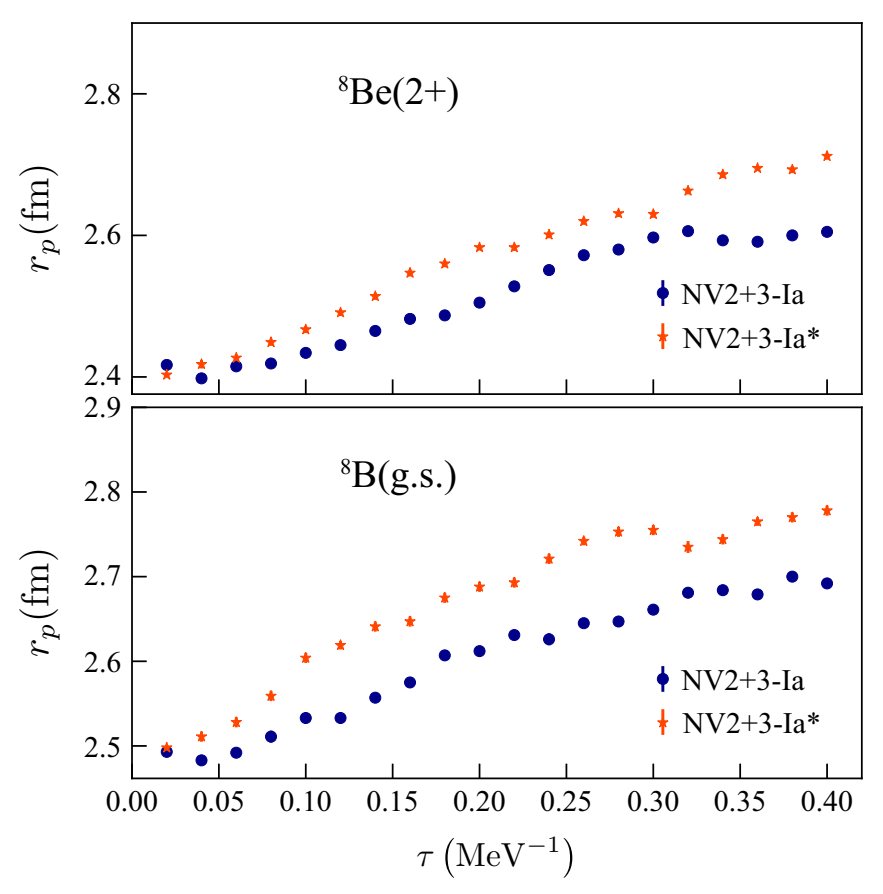

FIG. 3. GFMC propagation of the point-proton radius of the first excited state of ${ }^{8} \mathrm{Be}$ (upper panel) and ${ }^{8} \mathrm{~B}$ (lower panel) based on the NV2+3-Ia and NV2+3-Ia* Hamiltonians.

imaginary time interval selected for the averaging. We find that such a procedure generates an additional uncertainty of $\approx 5 \%$ which is added in quadrature to the statistical one, and quoted in Table VI below of GFMC results.

\section{A. Weak transitions in $A=6-10$ nuclei}

In this section, we present results for the GT reduced matrix element (RME) defined as

$$
\mathrm{RME}=\frac{\sqrt{2 J_{f}+1}}{g_{A}} \frac{\left\langle J_{f} M\left|j_{5, \pm}^{z}\right| J_{i} M\right\rangle}{\left\langle J_{i} M, 10 \mid J_{f} M\right\rangle},
$$

where $j_{5, \pm}^{z}$ is the $z$ component (at vanishing momentum transfer) of the charge-raising/lowering current with $\mathbf{j}_{5, \pm}=\mathbf{j}_{5, x} \pm$ $i \mathbf{j}_{5, y}$, and $\left\langle J_{i} M, 10 \mid J_{f} M\right\rangle$ is a Clebsch-Gordan coefficient.

Results for the GT RMEs in $A=6-10$ nuclei based on variational wave functions are reported in Table $\mathrm{V}$ for the eight different NV2+3 interactions discussed above, namely the NV2+3-Ia/b, NV2+3-IIa/b, and corresponding starred models. The one-body axial current at LO, illustrated in Fig. 1(a), leads to contributions to the matrix elements reported in the third column of Table V. One-body relativistic corrections (N2LO-RC) and two-body currents of one-pion range (N2LO- $\Delta$ ) at N2LO, displayed in Figs. 1(b) and 1(c), are added up and given in the fourth column of Table $\mathrm{V}$ labeled with $\mathrm{N} 2 \mathrm{LO}-(\mathrm{RC}+\Delta)$. A rough estimate of the size of the RC corrections can be obtained suppressing each LO term by a factor of $(Q / m)^{2} \approx 0.01$, where we used a "typical" nucleon's low-momentum $Q \approx 100 \mathrm{MeV}$. Contributions at N3LO are given in the columns labeled by N3LO-OPE and N3LO-CT, corresponding to the one-pion range and contact currents [and displayed in Figs. 1(d) and 1(e)]. The cumulative contributions are given in the next to last column, while the contributions beyond LO only in the column labeled "TotalLO." Experimental data from Refs. [75-79] are reported in the last column of Table $\mathrm{V}$.

All the calculations use axial currents at tree-level which are consistent with the specific NV2+3 model used to generate the VMC wave functions. VMC results based on different nuclear Hamiltonians are qualitatively in agreement. In particular, for the $A=3,6,7$, and 10 systems the LO contribution provides about $97 \%$ of the total matrix elements with currents beyond LO giving the remaining $\lesssim 3 \%$ correction. This correction adds up constructively to the LO contribution for all nuclei being considered, but for the $A=10$ transition. For this last transition, we find that the contributions beyond LO give a correction that quenches the LO results obtained with all the starred models, and with the unstarred NV2+3-IIb interaction. More details about this calculation will be given in the following section. We emphasize that the "Total-LO" column includes, in addition to two-body contributions, also a small correction resulting from the one-body N2LO-RC current.

Transitions involving $A=8$ nuclei exhibit a large suppression at LO. This behavior is attributable to the fact that the initial and final VMC wave functions are characterized by different dominant spatial symmetries, which make their overlap small compared to cases in which both the initial and final states display the same dominant spatial symmetry. As a consequence, in these cases the LO term is only about $\approx 40-50 \%$ of the total matrix element, with two-body currents providing a large correction. Two-body currents, while improving the agreement with the experimental values, are insufficient to fully explain them. Because of the reduced overlap between dominant components in the wave functions, these matrix elements are particularly sensitive to small components, which are poorly constrained and model dependent. This can be appreciated by looking at the one-body transition densities, $\rho^{1 \mathrm{~b}}\left(r_{i}\right)$, defined as

$$
\mathrm{RME}(1 \mathrm{~b})=\mathrm{RME}(\mathrm{LO})=4 \pi \int d r_{i} r_{i}^{2} \rho^{1 \mathrm{~b}}\left(r_{i}\right),
$$

where $r_{i}$ is the distance of nucleon $i$ from the center-of-mass of the system.

In Figs. 4 and 5, we show one-body densities for two transitions, namely the $\epsilon$ capture of the ${ }^{7} \mathrm{Be}$ ground state to the ${ }^{7} \mathrm{Li}$ ground state and the ${ }^{8} \mathrm{Li} \beta$ decay. The former, involves initial and final states with the same [43] (dominant) spatial symmetry, while for the latter the initial state is in a [431] spatial symmetry configuration and the final state is in a [44] one. The densities are calculated using the NV2+3-Ia/b and NV2+3-IIa/b interactions. From the figures we can see that the $A=7$ one-body densities are well constrained and essentially model independent, while the $A=8$ ones are particularly sensitive to the nuclear Hamiltonian used to generate the wave functions. Of course, these considerations are based on VMC results. A GFMC propagation might mitigate the observed model dependence.

In all the cases we studied, the contactlike current at N3LO provides a correction that quenches the LO terms, while the currents of one-pion range at N2LO and N3LO add up 
TABLE V. Gamow-Teller RMEs in $A=6,7,8$, and 10 nuclei obtained with chiral axial currents [4] and VMC wave functions corresponding to the NV2+3-Ia/b and NV2+3-IIa/b (NV2+3-Ia/b* and NV2+3-IIa/b*) Hamiltonian models [1,2,4,37]. Columns labeled with LO, N2LO-(RC+ $\Delta)$, N3LO-OPE, and N3LO-CT refer to the contributions given by the diagrams illustrated in Figs. 1(a) and 1(b) plus Figs. 1(c), 1(d) and 1(e), respectively. The cumulative results are reported in the column labeled "Total," while results including only corrections beyond LO are listed under "Total-LO." Experimental values from Refs. [75-79] are given in the last column. The dominant spatial symmetries of the VMC wave functions are reported in the first column. Statistical errors associated with the Monte Carlo integrations are not shown but are below $1 \%$.

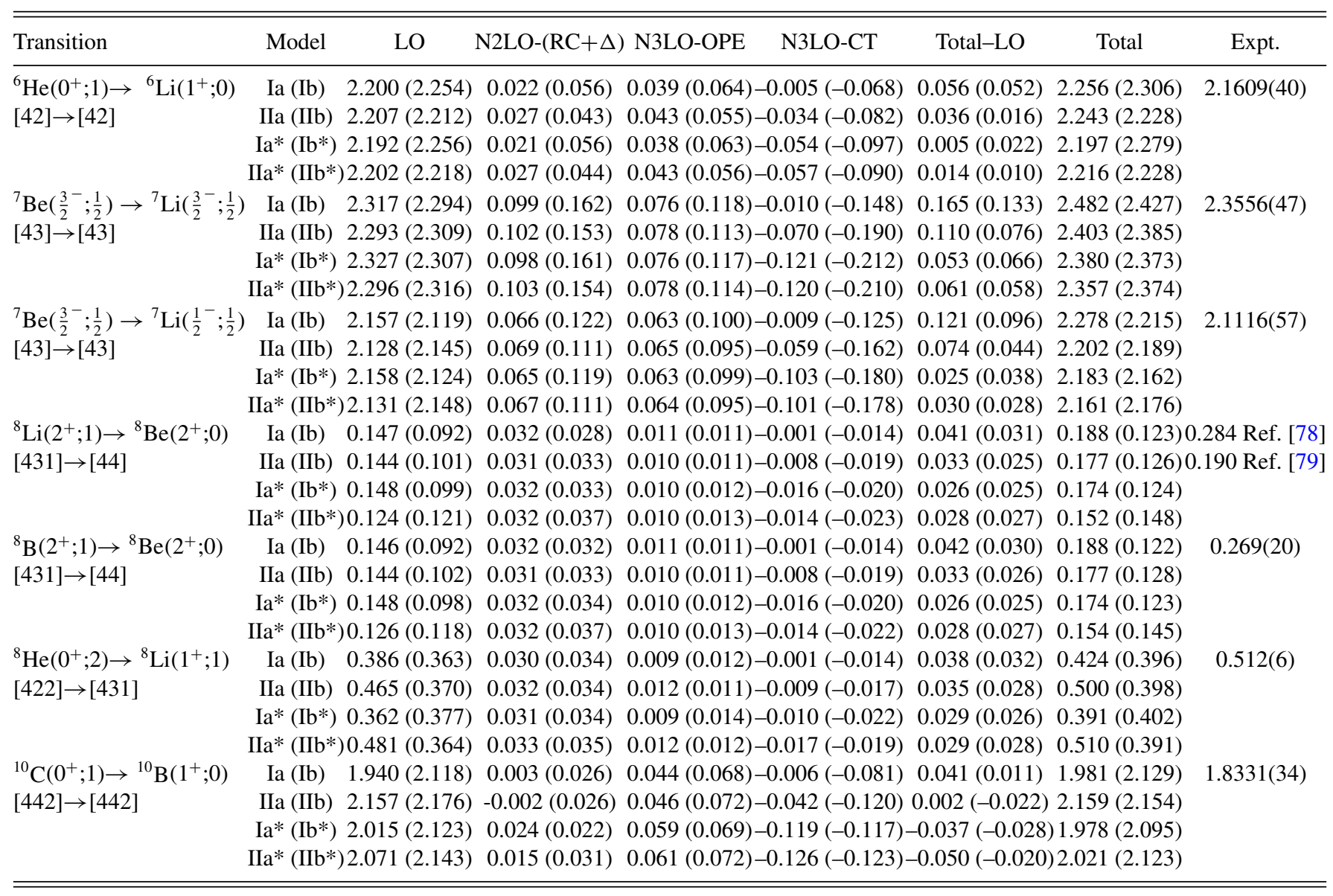

constructively to the LO contributions (see Table V). The main difference between the un-starred and starred calculations is observed in the size of the contact contribution. In particular,

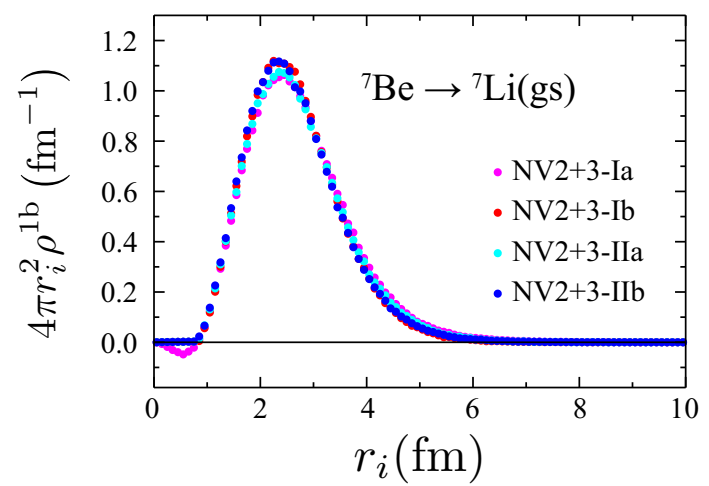

FIG. 4. One-body density-defined in Eq. (18)—of the ${ }^{7} \mathrm{Be}$ to ${ }^{7} \mathrm{Li}(\mathrm{gs})$ GT RME obtained with models NV2+3-Ia/b and NV2+3$\mathrm{IIa} / \mathrm{b}$ (see text for explanation). starred models are characterized by a larger value of $z_{0}$ (see Table III), which in turns leads to a larger (in magnitude) N3LO-CT correction.

We performed GFMC propagations only for the NV2+3Ia and $\mathrm{NV} 2+3-\mathrm{Ia}^{*}$ models. GFMC results are reported in

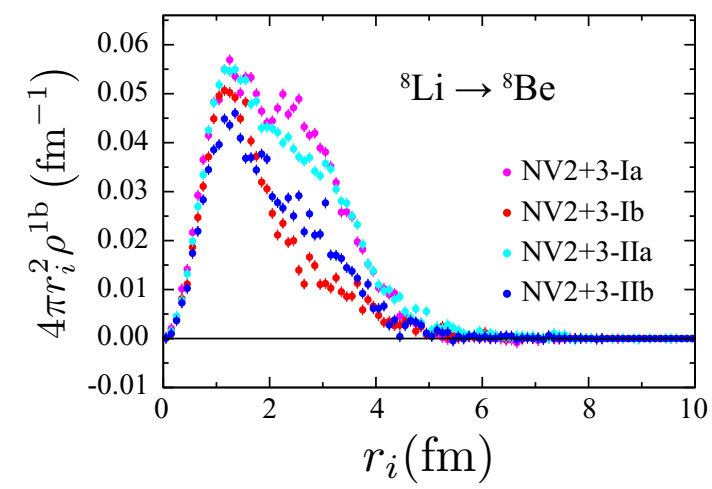

FIG. 5. Same as Fig. 4 but for the ${ }^{8} \mathrm{Li}$ to ${ }^{8} \mathrm{Be}$ GT RME. 
TABLE VI. Gamow-Teller RMEs in $A=6,7,8$, and 10 nuclei obtained with chiral axial currents [4] and GFMC (VMC) wave functions corresponding to the NV2+3-Ia and NV2+3-Ia* Hamiltonian models [1,2,4,37]. Results corresponding to the one-body current at LO (column labeled "LO"), and to the sum of all the corrections beyond LO (column labeled "Total-LO") are given, along with the cumulative contributions (column labeled "Total") to be compared with the experimental data [75-79] reported in the last row. Results from Ref. [6] based on the AV18+Il7 nuclear Hamiltonian are also shown where available. Statistical errors associated with the Monte Carlo integrations are not shown, but are below $1 \%$. Transitions to ${ }^{8} \mathrm{Be}$ are affected by an additional systematic error of $\approx 5 \%$, see text for explanation.

\begin{tabular}{|c|c|c|c|c|c|c|}
\hline Transition & Model & s.s. & LO & Total-LO & Total & Expt. \\
\hline${ }^{6} \mathrm{He}\left(0^{+} ; 1\right) \rightarrow{ }^{6} \mathrm{Li}\left(1^{+} ; 0\right)$ & $\mathrm{Ia}^{*}$ & & $2.107(2.192)$ & $0.011(0.005)$ & $2.118(2.197)$ & \\
\hline \multirow{3}{*}{${ }^{7} \mathrm{Be}\left(\frac{3}{2}^{-} ; \frac{1}{2}\right) \rightarrow{ }^{7} \mathrm{Li}\left(\frac{3}{2}^{-} ; \frac{1}{2}\right)$} & Ia & {$[43] \rightarrow[43]$} & $2.273(2.317)$ & $0.164(0.165)$ & $2.440(2.482)$ & $2.3556(47)$ \\
\hline & $\mathrm{Ia}^{*}$ & & $2.286(2.327)$ & $0.052(0.053)$ & $2.338(2.380)$ & \\
\hline & Ref. [6] & & $2.294(2.334)$ & $0.061(0.050)$ & $2.355(2.384)$ & \\
\hline & $\mathrm{Ia}^{*}$ & & $2.061(2.158)$ & $0.009(0.025)$ & $2.070(2.183)$ & \\
\hline & Ref. [6] & & $2.083(2.150)$ & $0.046(0.046)$ & $2.129(2.196)$ & \\
\hline \multirow[t]{2}{*}{${ }^{8} \mathrm{Li}\left(2^{+} ; 1\right) \rightarrow{ }^{8} \mathrm{Be}\left(2^{+} ; 0\right)$} & $\mathrm{Ia}$ & {$[431] \rightarrow[44]$} & $0.074(0.147)$ & $0.029(0.041)$ & $0.103(0.188)$ & 0.284 Ref. [78] \\
\hline & Ia* & & $0.096(0.148)$ & $0.025(0.026)$ & $0.120(0.174)$ & 0.190 Ref. [79] \\
\hline \multirow[t]{2}{*}{${ }^{8} \mathrm{~B}\left(2^{+} ; 1\right) \rightarrow{ }^{8} \mathrm{Be}\left(2^{+} ; 0\right)$} & Ia & {$[431] \rightarrow[44]$} & $0.091(0.146)$ & $0.035(0.042)$ & $0.125(0.188)$ & $0.269(20)$ \\
\hline & $\mathrm{Ia}^{*}$ & & $0.102(0.148)$ & $0.024(0.026)$ & $0.126(0.174)$ & \\
\hline${ }^{10} \mathrm{C}\left(0^{+} ; 1\right) \rightarrow{ }^{10} \mathrm{~B}\left(1^{+} ; 0\right)$ & Ref. [6] & & $2.032(2.062)$ & $0.016(0.015)$ & $2.048(2.077)$ & \\
\hline
\end{tabular}

Table VI, where, for completeness, we also show the corresponding VMC values in parentheses along with the GFMC results from Ref. [6]. We summarize the GFMC results in



FIG. 6. Ratios of GFMC to experimental values of the GT RMEs in the ${ }^{3} \mathrm{H},{ }^{6} \mathrm{He},{ }^{7} \mathrm{Be},{ }^{8} \mathrm{~B},{ }^{8} \mathrm{Be},{ }^{8} \mathrm{He}$, and ${ }^{10} \mathrm{C}$ weak transitions. Theory predictions correspond to the $\chi \mathrm{EFT}$ axial current at $\mathrm{LO}$ (empty symbols) and up to N3LO (filled symbols) obtained with the NV2+3-Ia and NV2+3-Ia* models. Results from Ref. [6] based on the AV18+IL7 nuclear Hamiltonian and N4LO currents from Ref. [5] are also shown. Results for the ${ }^{3} \mathrm{H}$ weak transition were reported in Ref. [4].
Fig. 6 and compare them (where possible) to the results of Ref. [6] based on the AV18+IL7 nuclear Hamiltonian.

The effect of the GFMC propagation in imaginary time is to reduce the $\mathrm{VMC}$ results by $\lesssim 4 \%$ in all selected transitions (but for the $A=10$ transition obtained with the NV2+3-Ia* model). The agreement with the data, after the inclusion of two-body currents, is at the $\approx 2 \%(\lesssim 2 \%)$ level for the $A=$ 6 transition with the NV2+3-Ia (NV2+3-Ia*) model; and at the $\lesssim 4 \%(\lesssim 1 \%)$ level for the $A=7$ cases with the NV2+3Ia (NV2+3-Ia*) model. These results are in agreement with those obtained for the same transitions in the calculations of Ref. [6] which were based on the AV18+IL7 interactions. The $\mathrm{NV} 2+3$ models lead to a more satisfactory agreement with the data for the $A=6 \mathrm{RME}$ primarily because, with these interactions, the LO term is $2 \%$ smaller than obtained using AV18+IL7 model.

The largest discrepancy generated by the use of different nuclear Hamiltonians, including AV18+IL7, is observed in the $A=10$ transition. This can be appreciated looking at both Table V and Table VI. From the former, we observe a rather large cutoff dependence (models a vs. models b), and also a large sensitivity to the class (either I or II) used to generate the nuclear wave functions. From Table VI, we see that the results of Ref. [6], based on the AV18+IL7 Hamiltonian, lie between models Ia and Ia*. This large model and cutoff dependence can be traced back to the existence of two nearby $J^{\pi}=1^{+}$ excited states in ${ }^{10} \mathrm{~B}$, the lower one a predominantly ${ }^{3} S_{1}[442]$ state and the upper one a ${ }^{3} D_{1}$ [442] state (in $L S$ coupling), which are only $1 \mathrm{MeV}$ apart. The transition from the ${ }^{10} \mathrm{C}\left(0^{+}\right)$ state, which is predominantly ${ }^{1} S_{0}$ [442], is large in the $S \rightarrow$ $S$ components, but about five times smaller in the $S \rightarrow D$ components. This makes the GT matrix element particularly 
sensitive to the exact mixing of the ${ }^{3} S_{1}$ and ${ }^{3} D_{1}$ components in the two ${ }^{10} \mathrm{~B}\left(1^{+}\right)$states produced by a given Hamiltonian. It would appear that none of the interactions models studied here gets quite the right mixing of these components. In particular, results based on the $\mathrm{NV} 2+3-\mathrm{Ia}$ and $\mathrm{NV} 2+3-\mathrm{Ia} *$ interactions overpredict the data by $\sim 7 \%$ and $12 \%$, respectively, which gives an indication of the spread of the theoretical estimates.

Predictions for the RMEs of $A=8$ transitions are the first QMC calculations for these systems that include corrections from two-body axial currents. As discussed above, RMEs are suppressed at leading order which gives only $\approx 50 \%$ and $\approx 40 \%$ of the experimental values for the ${ }^{8} \mathrm{~B} \rightarrow{ }^{8} \mathrm{Be}$ and ${ }^{8} \mathrm{He} \rightarrow{ }^{8} \mathrm{Li}$ transitions. Two-body currents provide about 20 $30 \%$ correction in the right direction which is, however, still insufficient to reach agreement with the experimental data. These transitions are challenging not only from the theoretical but also from the experimental point of view. For example, in Tables V and VI we quote two results for the RME of the ${ }^{8} \mathrm{Li} \rightarrow{ }^{8} \mathrm{Be}$ decay obtained from the $\log (f t)$ values of Ref. [78] and Ref. [79] via the following formula [77]

$$
\mathrm{RME}(\mathrm{EXPT})=\frac{1}{g_{A}} \sqrt{2 J_{i}+1} \sqrt{\frac{6139 \pm 7}{f t}},
$$

where $J_{i}$ is the angular momentum of the parent nucleus. The Fermi transition strength is small enough in this case that it can be neglected in the above formula. We then obtain two values, namely, RME (Ref. [78]) =0.284, and RME $($ Ref. [79] $)=0.190$. We note that Refs. [76,80], report a different overall factor of 6147 instead of 6139 in the formula given above. In our estimate we used $g_{A}=1.2723$. Despite this additional uncertainty in the deduced experimental values, our predictions still severely underestimate the data. For example, the calculated Ia* RME provides only $\approx 40 \%$ and $\approx 60 \%$ of the experimental values given in Ref. [78] and Ref. [79], respectively.

\section{B. Two-body transition densities}

In order to have a better understanding of the two-body terms in the axial current and their contributions, it is helpful to study the associated two-body transition densities, which we define as [81]

$$
\operatorname{RME}(2 \mathrm{~b})=4 \pi \int_{0}^{\infty} d r r^{2} \rho^{2 \mathrm{~b}}(r),
$$

where $r$ is the interparticle distance, and $2 \mathrm{~b}=\mathrm{N} 2 \mathrm{LO}-\Delta$, N2LO-OPE, and N2LO-CT.

Two-body transition densities, calculated for selected nuclei with variational wave functions corresponding to the Hamiltonian models NV2+3-Ia (Ia) and NV2+3-Ia* (Ia*), are presented in Fig. 7. These models produce similar N2LO$\Delta$ and N3LO-OPE densities, since they are based on the same underlying NV2 interaction and only differ in the NV3 interaction. Specifically, what differs is the strength $z_{0}$ of the contact current-linked to the (contact) three-nucleon interaction via the relation in Eq. (16). Because of the different methodologies adopted in constraining $c_{D}$ and $c_{E}$ (the LECs parametrizing the contact piece of the three-nucleon interaction), $z_{0}$ turns out to be much larger for Ia* than for Ia, see Table III. Since the contact current is proportional to $z_{0}$, this also explains why the corresponding density for Ia* is much larger (in magnitude) than for Ia. Note that they are both negative. As a consequence, the total density (black symbols in Fig. 7) develops a node at around $1 \mathrm{fm}$ in the case of model Ia*.

Another interesting feature of Fig. 7 is the difference between the N2LO- $\Delta$ and N3LO-OPE densities at separations $r \gtrsim 2 \mathrm{fm}$ for the transitions in the larger systems, especially those involving the $A=8$ resonant states. In the limit of vanishing momentum transfer we are considering here, the corresponding currents have the same operator structure [4], up to a momentum dependent term, absent in the N2LO- $\Delta$ current, which, however, we have explicitly verified to give a numerically small contribution by direct calculation. Examination of Eqs. (2.9) and (2.10) of Ref. [4] shows that this common operator structure involves two independent correlations functions $I^{(1)}\left(\mu_{i j} ; \alpha_{p}\right)$ and $I^{(2)}\left(\mu_{i j} ; \alpha_{p}\right)$ with $\mu_{i j}=m_{\pi} r_{i j}$, proportional to different combinations of LECs, denoted by $\alpha_{1}\left(\alpha_{1}^{\Delta}\right)$ and $\alpha_{2}\left(\alpha_{2}^{\Delta}\right)$ in the $\operatorname{OPE}(\Delta)$ current, with

$$
\begin{aligned}
& \frac{\alpha_{1}^{\Delta}}{\alpha_{1}}=\frac{c_{4}^{\Delta}}{c_{4}+1 /(4 m)} \approx 0.89, \quad \frac{\alpha_{2}^{\Delta}}{\alpha_{2}}=\frac{c_{3}^{\Delta}}{c_{3}} \approx 3.6, \\
& \frac{\alpha_{1}^{\Delta}}{\alpha_{2}^{\Delta}}=-\frac{1}{4}, \quad \frac{\alpha_{1}}{\alpha_{2}} \approx-1.0,
\end{aligned}
$$

using the values in Tables I and II ( $m$ is the average nucleon mass). Here $c_{3}^{\Delta}=-h_{A}^{2} /\left(9 m_{\Delta N}\right)$ and $c_{4}^{\Delta}=h_{A}^{2} /\left(18 m_{\Delta N}\right)$. Indeed, the N2LO- $\Delta$ current reads

$$
\mathbf{j}_{\Delta}^{\mathrm{N} 2 \mathrm{LO}}=-\left(\boldsymbol{\tau}_{i} \times \boldsymbol{\tau}_{j}\right)_{a} \boldsymbol{\sigma}_{i} \times \mathbf{O}_{i j}^{(1)}-\tau_{j, a} \mathbf{O}_{i j}^{(2)}+(i \rightleftharpoons j),
$$

with

$$
\mathbf{O}_{i j}^{(p)}=I^{(1)}\left(\mu_{i j} ; \alpha_{p}^{\Delta}\right) \boldsymbol{\sigma}_{j}+I^{(2)}\left(\mu_{i j} ; \alpha_{p}^{\Delta}\right) \hat{\mathbf{r}}_{i j} \boldsymbol{\sigma}_{j} \cdot \hat{\mathbf{r}}_{i j} .
$$

A similar expression holds for $\mathbf{j}_{\pi}^{\mathrm{N} 3 \mathrm{LO}}$ with $\alpha_{p}$ replacing $\alpha_{p}^{\Delta}$. There are cancellations between the terms proportional to $\mathbf{O}_{i j}^{(1)}$ and $\mathbf{O}_{i j}^{(2)}$ in each of these currents, and these cancellations are sensitive to the values of the ratios $\alpha_{1}^{\Delta} / \alpha_{2}^{\Delta}$ and $\alpha_{1} / \alpha_{2}$, and to the overlap between the wave functions of the states involved in the transition.

To gain insight into how short-range physics impacts these weak transitions across different nuclei, we display in Fig. 8 the densities corresponding to the individual two-body contributions, each normalized as $4 \pi r^{2} \rho^{2 \mathrm{~b}}(r) /\left(4 \pi r^{2} \rho^{2 \mathrm{~b}}\right)_{\max }$, where $\left(4 \pi r^{2} \rho^{2 \mathrm{~b}}\right)_{\max }$ is the maximum attained value (in magnitude); so all curves peak at 1 . We also display the total densities and note that, since both a positive peak and a negative valley are present in this case, each curve is normalized so that the value of the positive peak is 1 .

The universal behavior exhibited by the N2LO- $\Delta$, N3LOOPE, and N3LO-CT densities is quite striking, as the curves corresponding to different nuclei and different Hamiltonian models, essentially overlap for $r \lesssim 1 / m_{\pi}$. (It is even more striking when the weighing $r^{2}$ factor is not included). Such behavior can be understood as follows.

In a charge-raising process the two-body weak transition operators primarily convert a $p n$ pair with total spin/isospin 

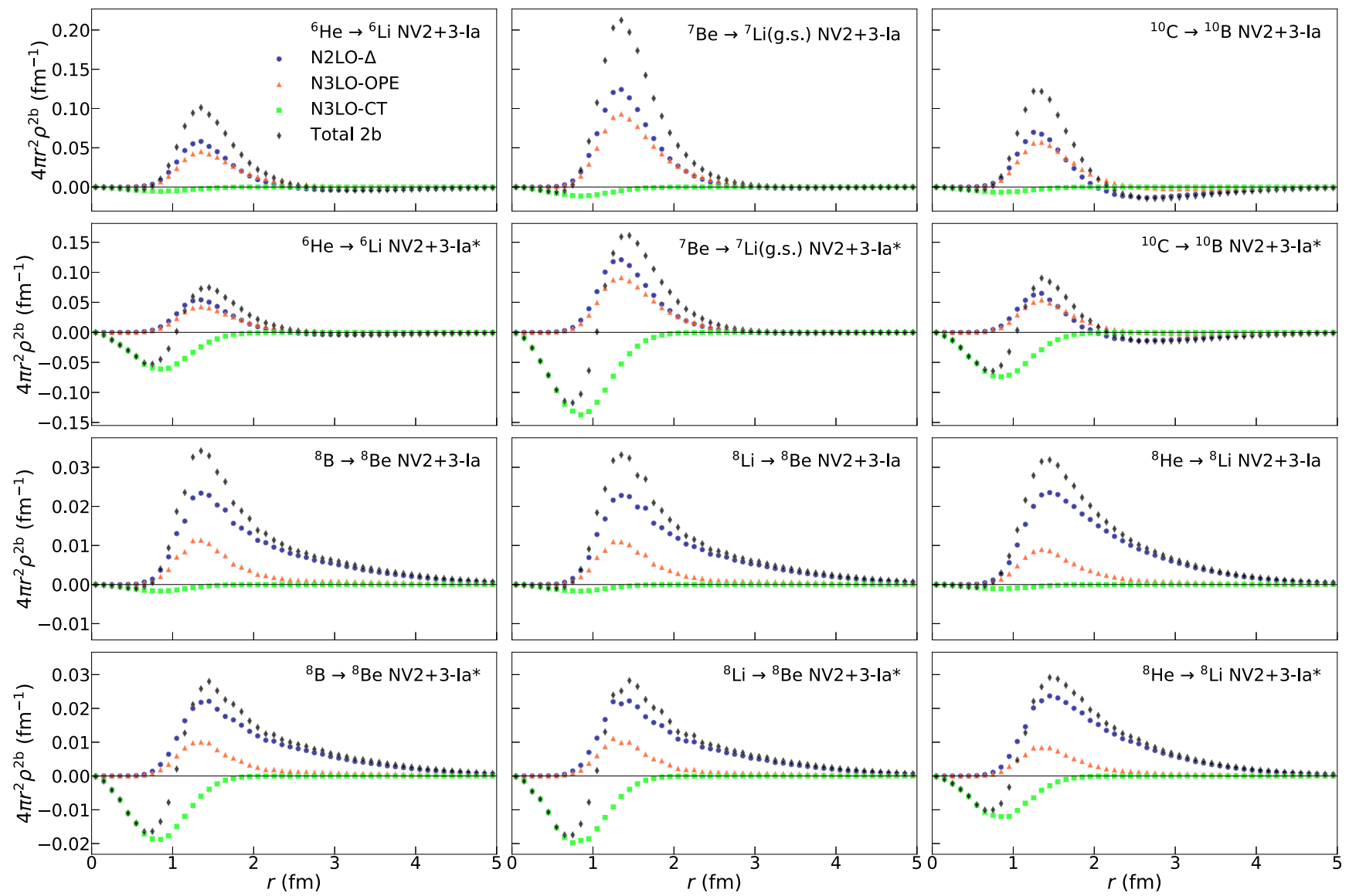

FIG. 7. Two-body transition density—see Eq. (20)—for selected nuclei obtained with the NV2+3-Ia and NV2+3-Ia* models (see text for explanation).


FIG. 8. Two-body transition density for selected nuclei (see text for explanation). Different colors indicate different transitions. Results obtained from calculations using the NV2+3-Ia model are represented with open circles and results obtained from calculations employing the $\mathrm{NV} 2+3-\mathrm{Ia}^{*}$ model results are represented by solid stars. 


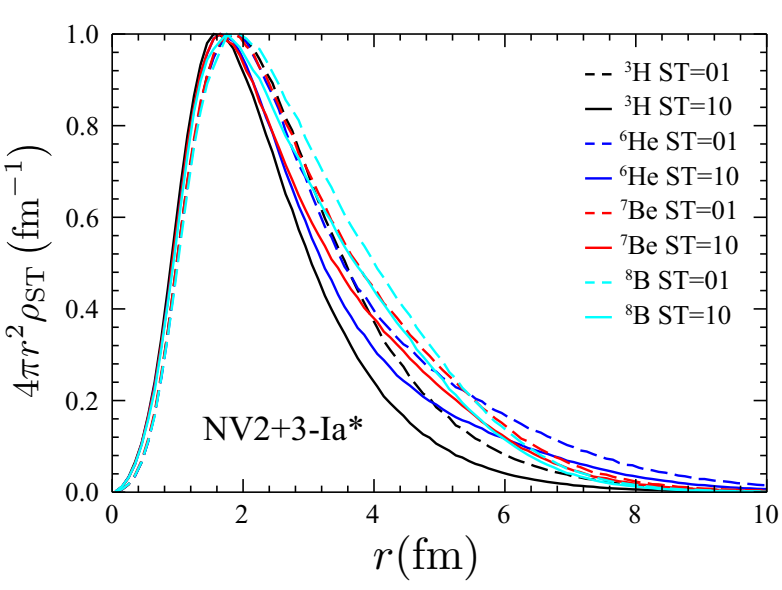

(a)

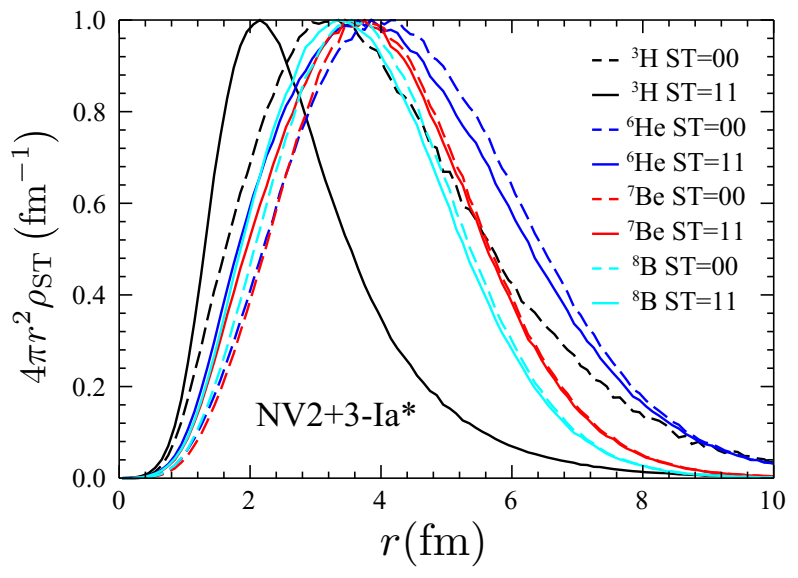

(b)

FIG. 9. Pair densities in (a) total spin/isospin $S / T=1 / 0$ and $0 / 1$ and (b) $S / T=0 / 0$ and $1 / 1$ obtained with VMC wave functions corresponding to model Ia*. All curves have been rescaled so as peak at 1 . Only central values are shown; statistical Monte Carlo errors are below a few percent.

$S / T=1 / 0$ (nn pair with $S / T=0 / 1)$ to a $p p$ pair with $S / T=0 / 1$ ( $p n$ pair with $S / T=1 / 0$ ) [81] - of course, similar considerations apply to a charge lowering process. These operators, at least in light systems, do not couple $T T_{z}=$ 10 and 00 to $T T_{z}=11$ in a significant way, since $P$-wave components are small in that case. At separations $\lesssim 1 / m_{\pi}$, where these transitions operators are most effective, the pair wave functions with $S / T=1 / 0$ and $0 / 1$ in different nuclei are similar in shape and only differ by a scale factor [82] - those corresponding to the Ia* model are illustrated in Fig. 9. This is the origin for the universal behavior observed in Fig. 8. Note that in Fig. 9 we also show the densities of pairs in $S / T=0 / 0$ and $1 / 1$, which do not scale. A complete analysis and interpretation of these results - in particular, of the role played by the tensor component of the nuclear interaction in shaping these densities - can be found in Ref. [82].

However, at separations $r \gtrsim 1 / m_{\pi}$, the N2LO- $\Delta$ and N3LO-OPE densities (especially the former) do not scale, particularly in the case of the heavier systems with $A \geqslant 8$, presumably due to delicate cancellations between the terms proportional to the operators $\mathbf{O}_{i j}^{(1)}$ and $\mathbf{O}_{i j}^{(2)}$ present in these currents $(P$-wave components in the wave functions of these systems may also play a significant role). As a matter of fact, because of the different long-range behavior present in the $A \geqslant 8$ transitions (note, e.g., how the N2LO- $\Delta$ density in the ${ }^{10} \mathrm{C}$ transition assumes negative values at large separations, all the other densities being positive), and because of the rather large size of the contact contribution in the Ia* model, the total densities exhibit nodes when calculated with the starred interactions, which leads to nontrivial cancellations.

\section{CONCLUSIONS}

In this work, we reported on a detailed study of weak matrix elements in $A=3-10$ systems based on chiral (two- and three-nucleon) interactions and associated (one- and two-body) axial currents at high orders in the chiral expansion. A summary of our results is displayed in Fig. 6. Agreement with the experimental data is obtained when correlated nu- clear wave functions are adopted. For these transitions the contribution of corrections beyond LO in the axial current is typically at the $\approx 2 \%$ level of the value calculated with the LO Gamow-Teller operator. These findings are in line with those reported in the hybrid study of Ref. [6] for the same transitions. Here, we also present calculations of matrix elements entering the rates of the ${ }^{8} \mathrm{Li},{ }^{8} \mathrm{~B}$, and ${ }^{8} \mathrm{He} \beta$ decays. These matrix elements are found to be suppressed at LO, and N2LO and N3LO currents provide a large correction $(\approx 20-30 \%)$ which is, however, insufficient to explain the experimental data. We attribute the large suppression at LO to the fact that the Gamow-Teller operator is, in these transitions, connecting large to small components of the initial and final wave functions. Improving on these calculations will require the development of more sophisticated wave functions with better constrained small components.

Finally, we also reported on a careful analysis of one- and two-body transition densities shown in Figs. 4-8. The latter are especially interesting because they allow us to understand the spatial distributions of the various two-body current operators, that is their behavior as functions of interparticle distance. We have shown that, for each set of interactions and consistent currents (either NV2+3-Ia or NV2+3-Ia*), the two-body transition densities exhibit a universal behavior at short distance across all nuclei we have considered in the present study.

\section{ACKNOWLEDGMENTS}

We thank V. Cirigliano, R. Charity, W. Dekens, J. Engel, A. Hayes, E. Mereghetti, and L. Sobotka for useful discussions at various stages of this work. S.P. and G.K. thank Grigor Sargsyan for useful discussions and for sharing information on the experimental $\log (f t)$ value of ${ }^{8} \mathrm{Li}$. The many-body calculations were performed on the parallel computers of the Laboratory Computing Resource Center, Argonne National Laboratory, and the computers of the Argonne Leadership Computing Facility (ALCF) via the 
2019/2020 ALCC grant "Low energy neutrino-nucleus interactions" for the project NNInteractions and via the 2020 INCITE grant " $A b$-initio nuclear structure and nuclear reactions". The work of J.C., S.G., and R.B.W. has been supported by the NUclear Computational Low-Energy Initiative (NUCLEI) SciDAC project. This research is supported by the
US Department of Energy, Office of Science, Office of Nuclear Physics, under contracts DE-AC05-06OR23177 (R.S.) and DE-AC02-06CH11357 (R.B.W.), DE-AC52-06NA25396 (S.G. and J.C.) and U.S. Department of Energy funds through the FRIB Theory Alliance award DE-SC0013617 (M.P. and S.P.).
[1] M. Piarulli, L. Girlanda, R. Schiavilla, R. N. Pérez, J. E. Amaro, and E. R. Arriola, Phys. Rev. C 91, 024003 (2015).

[2] M. Piarulli, L. Girlanda, R. Schiavilla, A. Kievsky, A. Lovato, L. E. Marcucci, S. C. Pieper, M. Viviani, and R. B. Wiringa, Phys. Rev. C 94, 054007 (2016).

[3] A. Baroni, L. Girlanda, A. Kievsky, L. E. Marcucci, R. Schiavilla, and M. Viviani, Phys. Rev. C 94, 024003 (2016); 95, 059902(E) (2017).

[4] A. Baroni et al., Phys. Rev. C 98, 044003 (2018).

[5] A. Baroni, L. Girlanda, S. Pastore, R. Schiavilla, and M. Viviani, Phys. Rev. C 93, 015501 (2016); 95 059901(E) (2017).

[6] S. Pastore, A. Baroni, J. Carlson, S. Gandolfi, S. C. Pieper, R. Schiavilla, and R. B. Wiringa, Phys. Rev. C 97, 022501 (2018).

[7] R. B. Wiringa, V. G. J. Stoks, and R. Schiavilla, Phys. Rev. C 51, 38 (1995).

[8] S. C. Pieper, AIP Conference Proceedings 1011, 143 (2008).

[9] P. Gysbers et al., Nat. Phys. 15, 428 (2019).

[10] D. Gazit, S. Vaintraub, and N. Barnea, in Proceedings of the 18th International Conference on Particles and Nuclei (PANIC'08) (Nuclear Physics A, 2009), pp. 1005-1007.

[11] A. Gardestig and D. R. Phillips, Phys. Rev. Lett. 96, 232301 (2006).

[12] D. Gazit, S. Quaglioni, and P. Navratil, Phys. Rev. Lett. 103, 102502 (2009); 122, 029901(E) (2019).

[13] R. Schiavilla (unpublished).

[14] M. Gonzalez-Alonso, O. Naviliat-Cuncic, and N. Severijns, Prog. Part. Nucl. Phys. 104, 165 (2019).

[15] J. Engel and J. Menendez, Rept. Prog. Phys. 80, 046301 (2017).

[16] J. Carlson, S. Gandolfi, F. Pederiva, S. C. Pieper, R. Schiavilla, K. E. Schmidt, and R. B. Wiringa, Rev. Mod. Phys. 87, 1067 (2015).

[17] J. E. Lynn, I. Tews, S. Gandolfi, and A. Lovato, Ann. Rev. Nucl. Part. Sci. 69, 279 (2019).

[18] R. B. Wiringa, Phys. Rev. C 43, 1585 (1991).

[19] B. S. Pudliner, V. R. Pandharipande, J. Carlson, S. C. Pieper, and R. B. Wiringa, Phys. Rev. C 56, 1720 (1997).

[20] K. M. Nollett, R. B. Wiringa, and R. Schiavilla, Phys. Rev. C 63, 024003 (2001).

[21] K. M. Nollett, Phys. Rev. C 63, 054002 (2001).

[22] N. Metropolis, A. W. Rosenbluth, M. N. Rosenbluth, A. H. Teller, and E. Teller, J. Chem. Phys. 21, 1087 (1953).

[23] M. Pervin, S. C. Pieper, and R. B. Wiringa, Phys. Rev. C 76, 064319 (2007).

[24] H. Krebs, E. Epelbaum, and U.-G. Meissner, Eur. Phys. J. A32, 127 (2007)

[25] R. N. Pérez, J. E. Amaro, and E. R. Arriola, Phys. Rev. C 88, 064002 (2013); Phys. Rev.C 91, 029901(E) (2015).

[26] R. Navarro Pérez, J. E. Amaro, and E. Ruiz Arriola, Phys. Rev. C 89, 024004 (2014).

[27] R. Navarro Perez, J. E. Amaro, and E. Ruiz Arriola, Phys. Rev. C 89, 064006 (2014).
[28] A. M. Green, Rept. Prog. Phys. 39, 1109 (1976).

[29] R. A. Arndt, R. L. Workman, and M. M. Pavan, Phys. Rev. C 49, 2729 (1994).

[30] V. G. J. Stoks, R. Timmermans, and J. J. de Swart, Phys. Rev. C 47, 512 (1993).

[31] M. Tanabashi, K. Hagiwara, K. Hikasa, K. Nakamura, Y. Sumino, F. Takahashi, J. Tanaka, K. Agashe, G. Aielli, C. Amsler et al. (Particle Data Group), Phys. Rev. D 98, 030001 (2018).

[32] U. van Kolck, Phys. Rev. C 49, 2932 (1994).

[33] E. Epelbaum, A. Nogga, W. Gloeckle, H. Kamada, Ulf G. Meissner, and H. Witala, Phys. Rev. C 66, 064001 (2002).

[34] J. E. Lynn, I. Tews, J. Carlson, S. Gandolfi, A. Gezerlis, K. E. Schmidt, and A. Schwenk, Phys. Rev. Lett. 116, 062501 (2016).

[35] I. Tews, S. Gandolfi, A. Gezerlis, and A. Schwenk, Phys. Rev. C 93, 024305 (2016).

[36] J. E. Lynn, I. Tews, J. Carlson, S. Gandolfi, A. Gezerlis, K. E. Schmidt, and A. Schwenk, Phys. Rev. C 96, 054007 (2017).

[37] M. Piarulli et al., Phys. Rev. Lett. 120, 052503 (2018).

[38] L. E. Marcucci, A. Kievsky, S. Rosati, R. Schiavilla, and M. Viviani, Phys. Rev. Lett. 108, 052502 (2012); 121, 049901(E) (2018).

[39] V. Cirigliano, W. Dekens, J. De Vries, M. L. Graesser, E. Mereghetti, S. Pastore, M. Piarulli, U. Van Kolck, and R. B. Wiringa, Phys. Rev. C 100, 055504 (2019).

[40] M. Piarulli and I. Tews, Front. Phys. 7, 245 (2020).

[41] S. Gandolfi, D. Lonardoni, A. Lovato, and M. Piarulli, Front. Phys. 8, 117 (2020).

[42] K. E. Schmidt and S. Fantoni, Phys. Lett. B 446, 99 (1999).

[43] B. D. Day, Rev. Mod. Phys. 39, 719 (1967).

[44] M. Baldo and G. F. Burgio, Rep. Progr. Phys. 75, 026301 (2012).

[45] S. Fantoni and S. Rosati, Nuovo Cimento A 25, 593 (1975).

[46] V. R. Pandharipande and R. B. Wiringa, Rev. Mod. Phys. 51, 821 (1979).

[47] M. Piarulli, I. Bombaci, D. Logoteta, A. Lovato, and R. B. Wiringa, Phys. Rev. C 101, 045801 (2020).

[48] I. Bombaci and D. Logoteta, Astron. Astrophys. 609, A128 (2018).

[49] T.-S. Park, D.-P. Min, and M. Rho, Phys. Rept. 233, 341 (1993).

[50] H. Krebs, E. Epelbaum, and U. G. Meißner, Ann. Phys. 378, 317 (2017).

[51] S. Pastore, R. Schiavilla, and J. L. Goity, Phys. Rev. C 78, 064002 (2008).

[52] S. Pastore, L. Girlanda, R. Schiavilla, M. Viviani, and R. B. Wiringa, Phys. Rev. C 80, 034004 (2009).

[53] S. Pastore, L. Girlanda, R. Schiavilla, and M. Viviani, Phys. Rev. C 84, 024001 (2011).

[54] S. Kolling, E. Epelbaum, H. Krebs, and U. G. Meissner, Phys. Rev. C 80, 045502 (2009).

[55] S. Kolling, E. Epelbaum, H. Krebs, and U. G. Meissner, Phys. Rev. C 84, 054008 (2011). 
[56] H. Krebs, E. Epelbaum, and U.-G. Meißner, Phys. Rev. C 101, 055502 (2020).

[57] S. Bacca and S. Pastore, J. Phys. G 41, 123002 (2014).

[58] M. Piarulli, L. Girlanda, L. E. Marcucci, S. Pastore, R. Schiavilla, and M. Viviani, Phys. Rev. C 87, 014006 (2013).

[59] R. Schiavilla, A. Baroni, S. Pastore, M. Piarulli, L. Girlanda, A. Kievsky, A. Lovato, L. E. Marcucci, S. C. Pieper, M. Viviani et al., Phys. Rev. C 99, 034005 (2019).

[60] N. Nevo Dinur, O. J. Hernandez, S. Bacca, N. Barnea, C. Ji, S. Pastore, M. Piarulli, and R. B. Wiringa, Phys. Rev. C 99, 034004 (2019).

[61] L. E. Marcucci, F. Gross, M. T. Pena, M. Piarulli, R. Schiavilla, I. Sick, A. Stadler, J. W. Van Orden, and M. Viviani, J. Phys. G 43, 023002 (2016).

[62] L. Girlanda, A. Kievsky, L. E. Marcucci, S. Pastore, R. Schiavilla, and M. Viviani, Phys. Rev. Lett. 105, 232502 (2010).

[63] S. Pastore, S. C. Pieper, R. Schiavilla, and R. B. Wiringa, Phys. Rev. C 87, 035503 (2013).

[64] V. M. Datar et al., Phys. Rev. Lett. 111, 062502 (2013).

[65] S. Pastore, R. B. Wiringa, S. C. Pieper, and R. Schiavilla, Phys. Rev. C 90, 024321 (2014).

[66] S. Pastore, J. Carlson, S. Gandolfi, R. Schiavilla, and R. B. Wiringa, Phys. Rev. C 101, 044612 (2020).

[67] S. Pastore, J. Carlson, V. Cirigliano, W. Dekens, E. Mereghetti, and R. B. Wiringa, Phys. Rev. C 97, 014606 (2018).

[68] V. Cirigliano, W. Dekens, J. De Vries, M. L. Graesser, E. Mereghetti, S. Pastore, and U. van Kolck, Phys. Rev. Lett. 120, 202001 (2018).
[69] X. B. Wang, A. C. Hayes, J. Carlson, G. X. Dong, E. Mereghetti, S. Pastore, and R. B. Wiringa, Phys. Lett. B 798, 134974 (2019).

[70] G. Audi, A. Wapstra, and C. Thibault, Nucl. Phys. A 729, 337 (2003).

[71] D. R. Tilley, C. M. Cheves, J. L. Godwin, G. M. Hale, H. M. Hofmann, J. H. Kelley, C. G. Sheu, and H. R. Weller, Nucl. Phys. A 708, 3 (2002).

[72] D. R. Tilley, J. H. Kelley, J. L. Godwin, D. J. Millener, J. E. Purcell, C. G. Sheu, and H. R. Weller, Nucl. Phys. A 745, 155 (2004).

[73] R. B. Wiringa, Phys. Rev. C 73, 034317 (2006).

[74] R. B. Wiringa, S. C. Pieper, J. Carlson, and V. R. Pandharipande, Phys. Rev. C 62, 014001 (2000).

[75] A. Knecht et al., Phys. Rev. C 86, 035506 (2012).

[76] T. Suzuki, R. Fujimoto, and T. Otsuka, Phys. Rev. C 67, 044302 (2003).

[77] W. T. Chou, E. K. Warburton, and B. A. Brown, Phys. Rev. C 47, 163 (1993).

[78] E. K. Warburton, Phys. Rev. C 33, 303 (1986).

[79] B. Pritychenko, E. Běták, M. Kellett, B. Singh, and J. Totans, Nucl. Instrum. Methods A 640, 213 (2011).

[80] J. C. Hardy and I. S. Towner, Phys. Rev. C 91, 025501 (2015).

[81] R. Schiavilla et al., Phys. Rev. C 58, 1263 (1998).

[82] J. L. Forest, V. R. Pandharipande, S. C. Pieper, R. B. Wiringa, R. Schiavilla, and A. Arriaga, Phys. Rev. C 54, 646 (1996). 